\title{
Robotic Fabrication as Catalysts for Emergent Topologies and Traditions: Nomadic Small Pavilions and Permanent Mega Structures in Kuwait
}

Hussain Dashti

Kuwait University

\begin{abstract}
This paper reviews tendencies and drives for future parametric computational design and robotic fabrication/construction automation. It sheds light on the local current impact of the computational paradigm and mass-customized robotic fabrication in Kuwait. This paper is intended to answer the following two questions: Is parametric design and robotic fabrication allowing for emergent architectural topologies? Is robotic fabrication a catalyst for legitimizing change in architectural traditions at a local level?

This has been experimented on two building scales. One with more ephemeral or transient nomadic pavilions, designed by the author, intended to demand our momentary attention, offering essential opportunities for research, experimentation, heuristic testing and prototyping - public delight and exposure. Though impermanent, these can even go so far as to be catalysts for positive change displaying affirmative qualities of temporal architecture.

On the other hand, the author shares parametric design and robotic fabrication practices/consultation on local permanent mega structures currently under construction. Such mega buildings act as proof that geometrically complex buildings do not stay in the realm of small experimental and heuristic research only, but incorporated in large-scale complex building, branding and placing countries on the global map.

Robotic fabrication and construction gives rise to new paradigms such as "zero-tolerance" building with "file-to-factory" production allowing for Ruskinian tectonics blending structures with ornamental aesthetics, similar to gothic architecture. With the profusion of robotic fabrication and construction, the author claims that change in the physical built environment is eminent. A final inquiry will be raised as a future research topic pertaining to robotic in-situ "mobility-on-demand", Artificial Intelligence, "Machine Learning", "Big Data" and "evolutionary robotics" which raises the question of what will our future mass-customized cities look like and what type of physical infrastructure is needed to facilitate mobile robotic fabrication and construction.
\end{abstract}

Keywords: Robotic fabrication; Mega structures; Nomad; Monad; Pavilion

\section{Introduction}

From a sustainable perspective, mass-production and consumption can produce a serious problem of natural resource deterioration. Until a short while ago, the objectives of architectural practices were, concentrating on the functional, structural, and aesthetic, (Utilitas, Firmitas, Venustas) character of architecture. More recently, we are concerned about efficiency and reasonably sustainable and harmoniously integrated in the indigenous environment.

During the eighteenth Century, Europeans philosophers attempted to tackle the question of Knowledge. There were two predominant counter arguments, on one hand, the rationalist trend, supported by 
Descartes and Spinoza, which stressed upon the manner of conceiving knowledge, where reason comes before experience strengthening the innate nature of things. On the other hand, empiricism, adopted by Hume and Locke, which supports knowledge with testimony of senses and experience. Empirical ways of seeking knowledge has been widely utilized in modernism based more on methods, experimentation has been consolidated, and the door has been opened to new digital technologies, and computational processes. Therefore, some concepts started to make sense such as emergence, complexity, and selforganization, which explain fundamental behavior and phenomena for contemporary science.

Digital computational technologies are currently ubiquitous and are definitely being utilized on our buildings and the analysis of our cities. For the last 20 years, the profound impact of computeraided techniques on architecture has been well charted. From the use of standard drafting packages to the more Avant-guard heuristic use of generative design tools and parametric modelling, digital technologies have come to play a major role in emergent building topologies and city layout helping architects to work both on buildings and urban scales changing the way in which we perceive, design, and build our structures based on unprecedented construction methods and techniques.

\section{Emergent Technology}

The emergent technologies and Design, develop skills, and help in pursuing knowledge in architectural design science that is located in new production paradigms. It continues to investigate new synergies of architecture and ecology through the critical intersection of computational design and digital fabrication; projects and products pursued by multiple iterations through hypothesis, material and computational experimentation, robotic fabrication, and evaluation. It involves experimenting with numeric data that makes good description of behavior, morphology, and material properties, that can cover numerous fields of design concentration including materials systems, advanced fabrication, natural and ecological systems design, algorithmic design of urban systems that can be embedded in algorithmic spatial models for culture, climate, economy, and ecology. Rapid production of Alternative are produced through measuring, quantifying, analyzing, and returned as input in the next generative series.

The tangible has caught up with the intangible, and possibilities that were entirely speculative in nonphysical domains just a few years ago are now being materialized and built. The term 'digital' no longer only conjures up notions of complex representation, computational geometries and intelligent systems, but also fabrication, craft and materials. At the same time, designers are faced with (and connected to) more tools to make their work than ever before, each packing extraordinary power, accuracy, capability and provocation. Subsequently, the scope on how these tools influence, validate or facilitate the designer is expanding rapidly and the challenge is to navigate such dynamic circumstances with a clear sense of critique on how they assist in advancing architecture as a visionary practice and subject.

\section{Emergence}

Emergence is a classical concept in systems theory, where it denotes the principle that the global properties defining higher order systems or "wholes" (e.g. boundaries, organization, control ...) can in general not be reduced to the properties of the lower order subsystems or "parts". Such irreducible properties are referred to as 'emergent.' Until now there is no satisfactory theory explaining what characterizes emergent properties

or what are the conditions for their existence. In this paper, I argue that computational design and robotic fabrication is allowing for emergent design processes and architecture cultures as catalysts for emergent architecture design culture as well as emergent physical topologies. During the last decades, we started observing new architectural typologies that are otherwise unachievable with conventional, non-computational and Non-robotized fabrication, which may include dynamic and spontaneous selforganizing emergent designs based on complex-related parameters. The spontaneous creation of 
an "organized whole" out of a "disordered" collection of interacting parts, as witnessed in selforganizing systems in physics, chemistry, biology, sociology ..., is a basic part of either simple or a more complex hierarchy of dynamic emergence (Haken 2014). This self-organizing emergence could be simultaneously hierarchical, characterized at two level structures: the "Microscopic" level, where elements interact, and the "macroscopic" level, where these interactions lead to global patterns varying in the level of complexity. If we wish to understand the architecture of such complexity, we will need a more general, integrating theory of emergence and self-organization. Rapid production of Alternatives are produced through measuring, quantifying, analyzing, and returned as input in the next generative series producing numerous derivatives and iterations that can be file-to-factoried to digital fabrication robots. This digital design/fabrication process is emerging into a new set of design culture, process, production protocols, and professional rolls, allowing architects to be involved in the 'making' production process, reducing the conventional role of the contractor.

\section{High Definition Zero Tolerance}

As an academic of architecture with interest in computational design and digital fabrication, I attempt to anticipate the natural proclivity and to look forward on how fecund new developments in technology might prove to architecture. Through design/build experimentation, I attempt to establish a deeper and sustained interest in fabrication focusing on computational design, digital fabrication, verification technologies, 3D scanning and its associated interface with modelling and manufacture.

Cutting edge technology is permitting high-definition zero-tolerance building with high degree of freedom in design pushing for mass-customized production processes. (Castles, 2014) This represents a significant paradigm shift in the area of design and fabrication process adopted both in academia through design pedagogy as well as in architectural practice. This paper focuses on the meaning of tolerance across conditions of fine-grain information, predominantly from a design perspective, but there is also the sense that we need to remain attentive of the opportunities that might be afforded, or lost, across practice and within the construction industry, as Helen Castle 2014, in her editorial for Architectural Design issue "High-Definition and Zero Tolerance" stated.

She provides a holistic introduction on the work of Achem Mengez and Bob Sheil at the AA (1982), using innovative technology with work from Tobias Nolte and Andrew Witt's (2010), description of the self-optimization system that Gehry Technologies developed for the realization of the Foundation Louis Vuitton art museum in Paris. Skylar Tibbits 2015, also provides an insightful account of the 4D printing processes that the Self-Assembly Lab at the Massachusetts Institute of Technology (MIT) is collaborating on with multi-material printing company.

\section{Nomadic Pavilions in Kuwait}

For architects, small-scale temporary structures offer essential opportunities for research and experimentation - for testing and prototyping - and to get their work built and known by a wider public. Though impermanent, the majority of structures are still realized with a function or purpose, whether cultural, commercial or social. These can even go so far as to be catalysts for positive change, as described by Dan Hill ('A Sketchbook for the City to Come: The Pop-Up as R\&D', pp 32-9) and Andrea Kahn ('Building Community', pp 72-9) at a local level, and Martyn Hook ('The Affirmative Qualities of a Temporal Architecture', pp 118-23) at the international scale. Interceding even with a provisional structure with social purpose offers the opportunity to provide and to act where it may not be possible or relevant to do so permanently. As Leon Describes pavilions as 'a fairground assemblage', highlighting both the wonder and the novelty of this constellation of 'new forms' emerging as part of 'an ancient lineage'. Like ancient fairs, these temporary structures are no sooner drawing us in with their amboyant originality as they are disbanding. This very ephemeral nature may apply on the local nomadic 
Bedouin tents in Kuwait where it is perhaps this that keeps us most enwrapped by them; you are never quite sure when they will be gone. This pavilion is a prototype catalyst for positive change utilizing emergent technology to create emergent ephemeral novel topology as illustrated in Figures (1, 2, and 3).
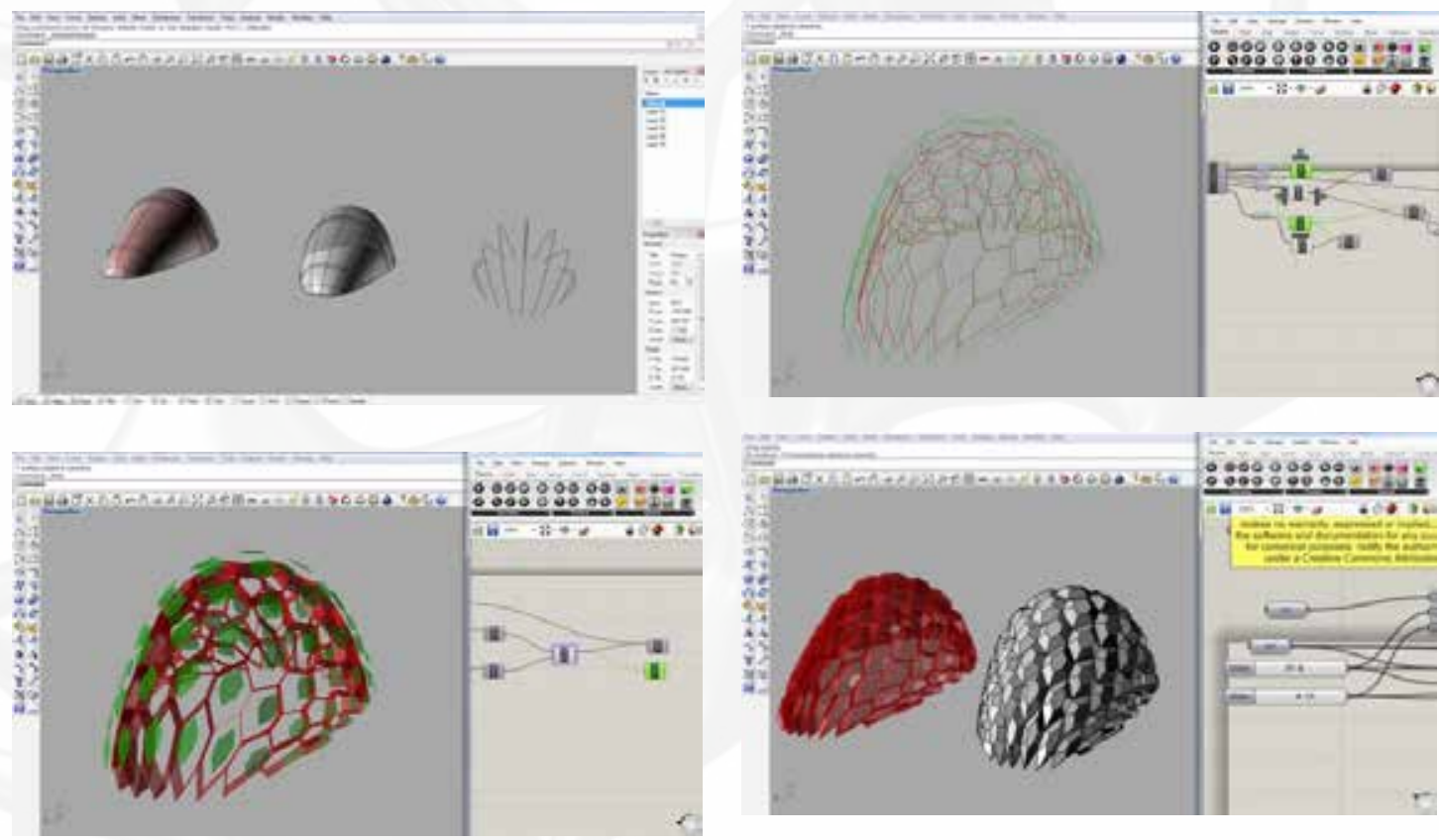

Figure 01: Parametric modeling of Pavilion 6.
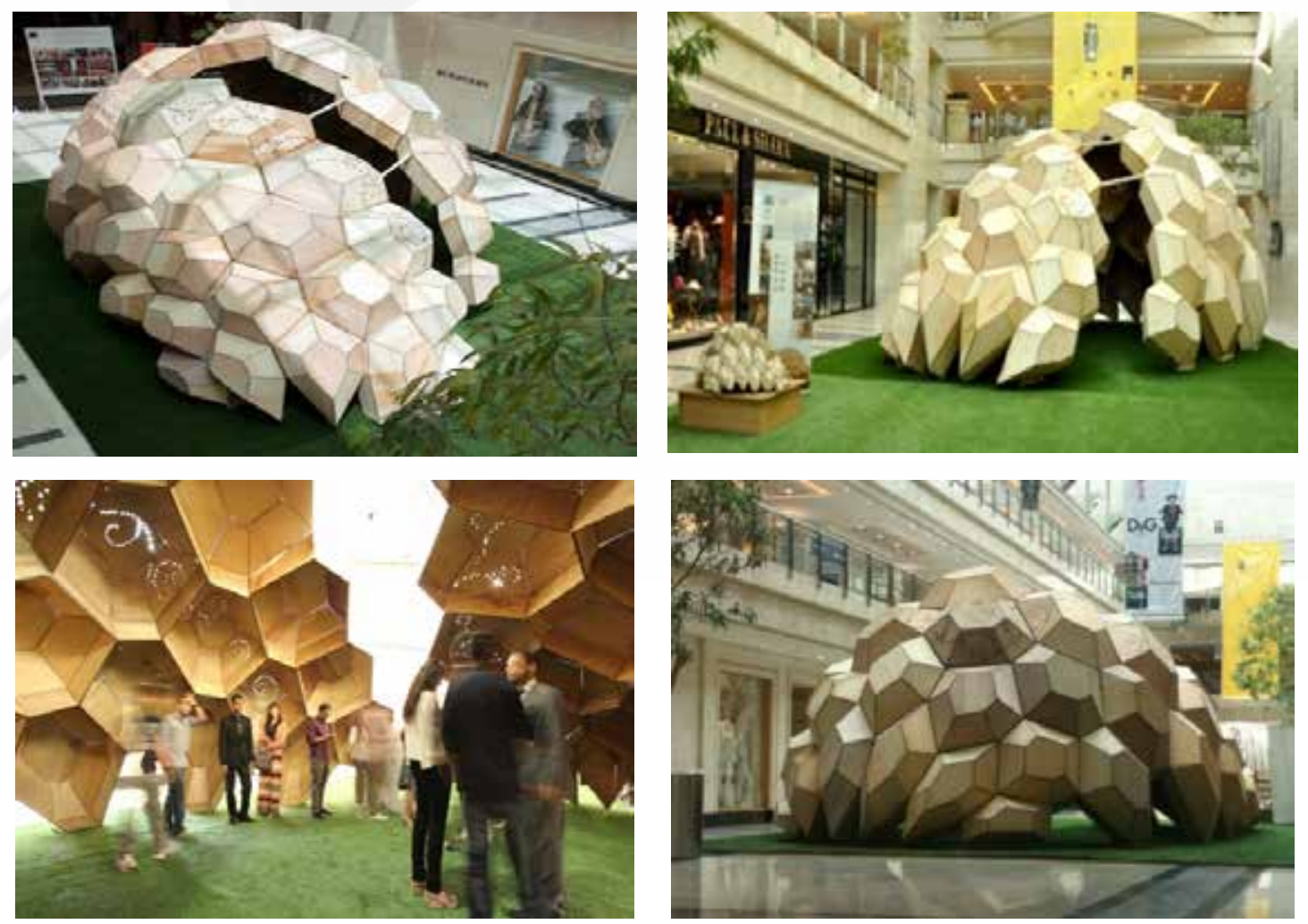

Figure 02: Design and build of Pavilion. 

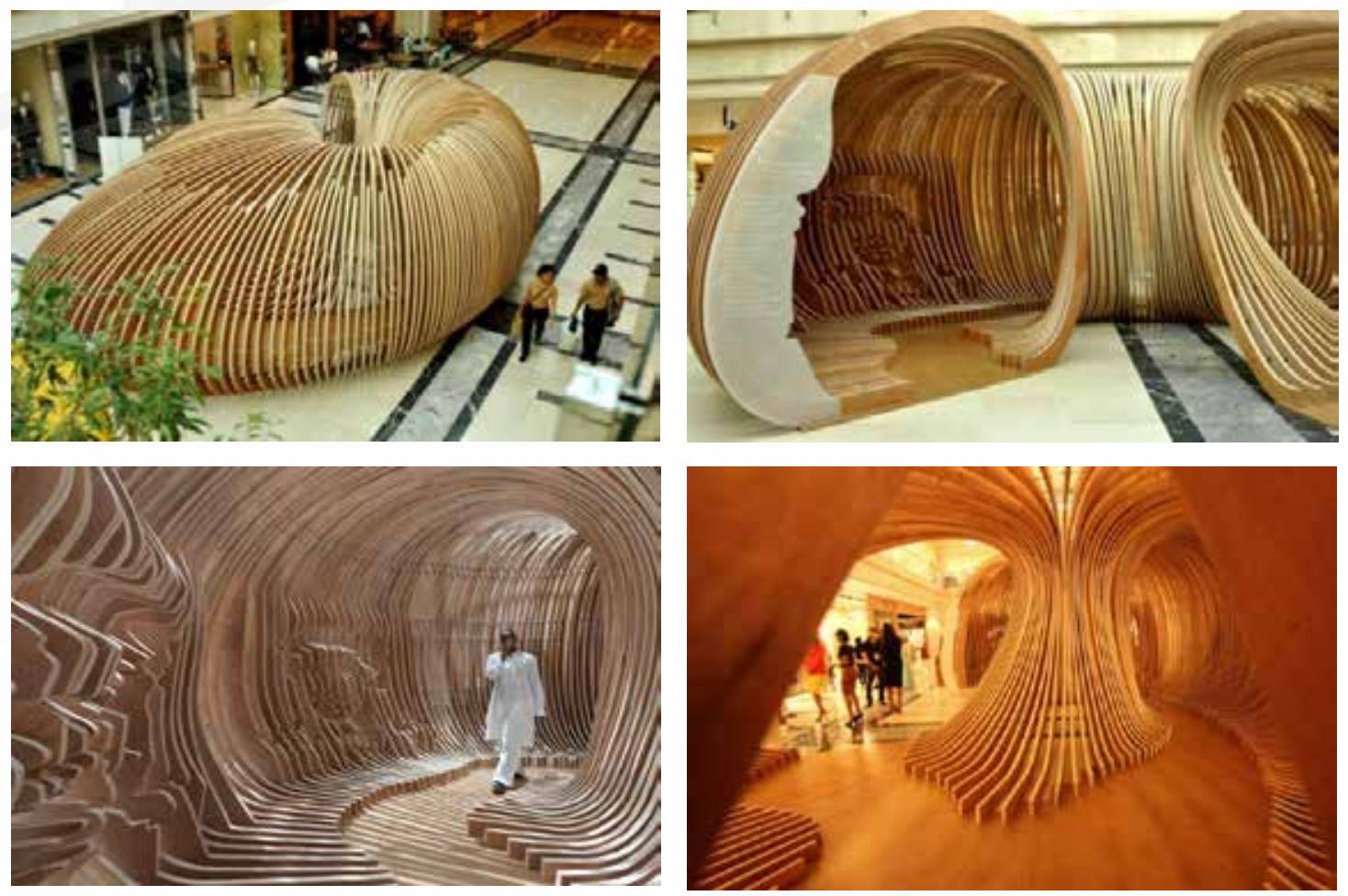

Figure 03: Design and build of OV Pavilion.

\section{Mega Structures in Kuwait}

The National Bank of Kuwait (NBK) and the new Kuwait airport, both designed by Norman Fosters Group, are prime examples of the emergent cutting-edge technology of computational design and robotic fabrication. The author is deeply aware of the design and fabrication of these two projects. Based on multi-parameters including structures, function, program requirement, environmental performance, building materials, and fabrication technology, it is evident that both buildings forms' were parametrically designed to simultaneously deal with the above mentioned numerous parameters. This created a high degree of complexity both as a design as well as construction and fabrication process. The high precision and low-tolerance in fabrication and construction of these two projects required such emergent technology that otherwise brought the construction to a halt; emergent fabrication technology such as robotic arms for mold making and finishing treatment, Robotic Adaptive Molds (RAM) enabling free form fabrication, dynamic laser scanning for surveying and shell-cast fabrication and assembly, as illustrated in Fig. $4-13$ below. I believe a new design culture is introduced to the local architecture design and fabrication practice. 

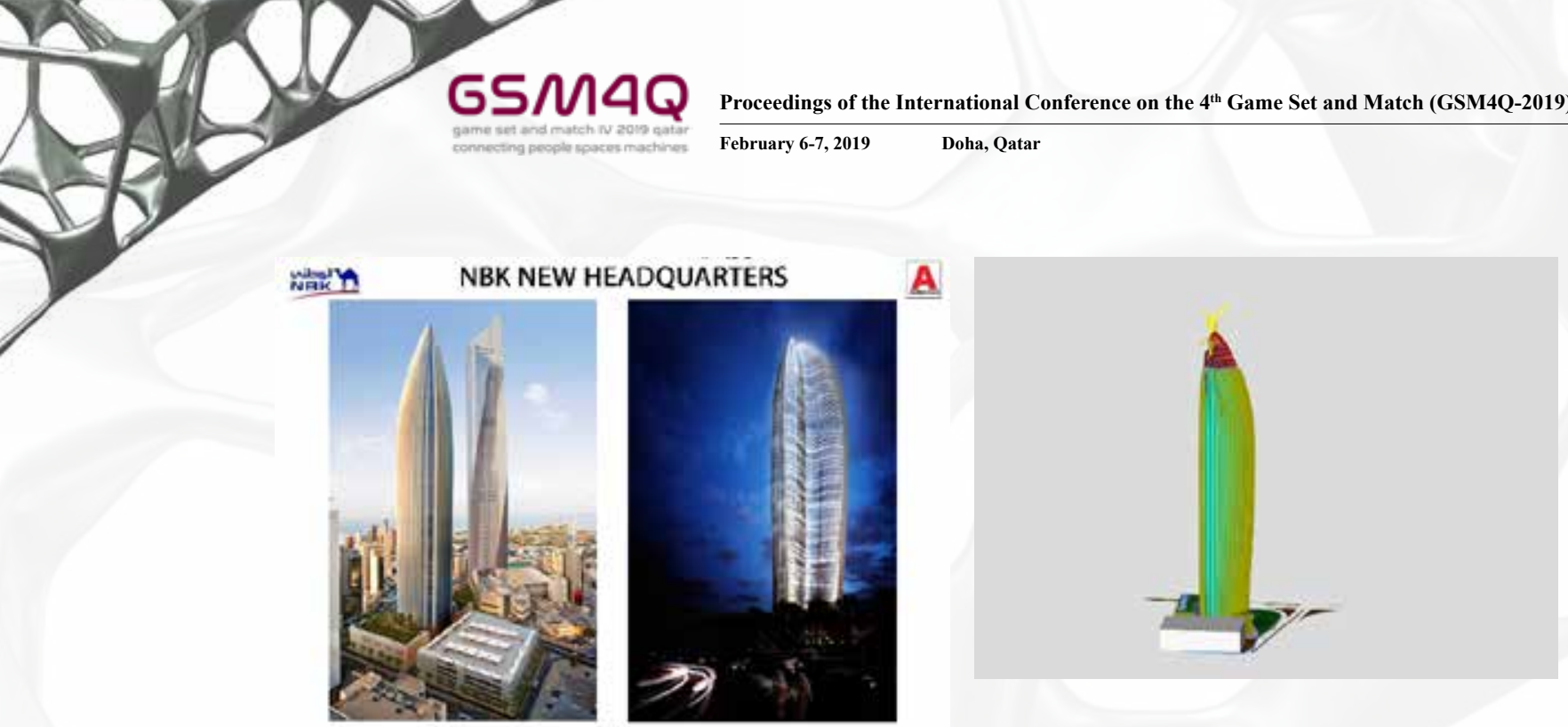

Figure 04: The new National Bank of Kuwait (NBK) headquarter designed by Norman Fosters Group.
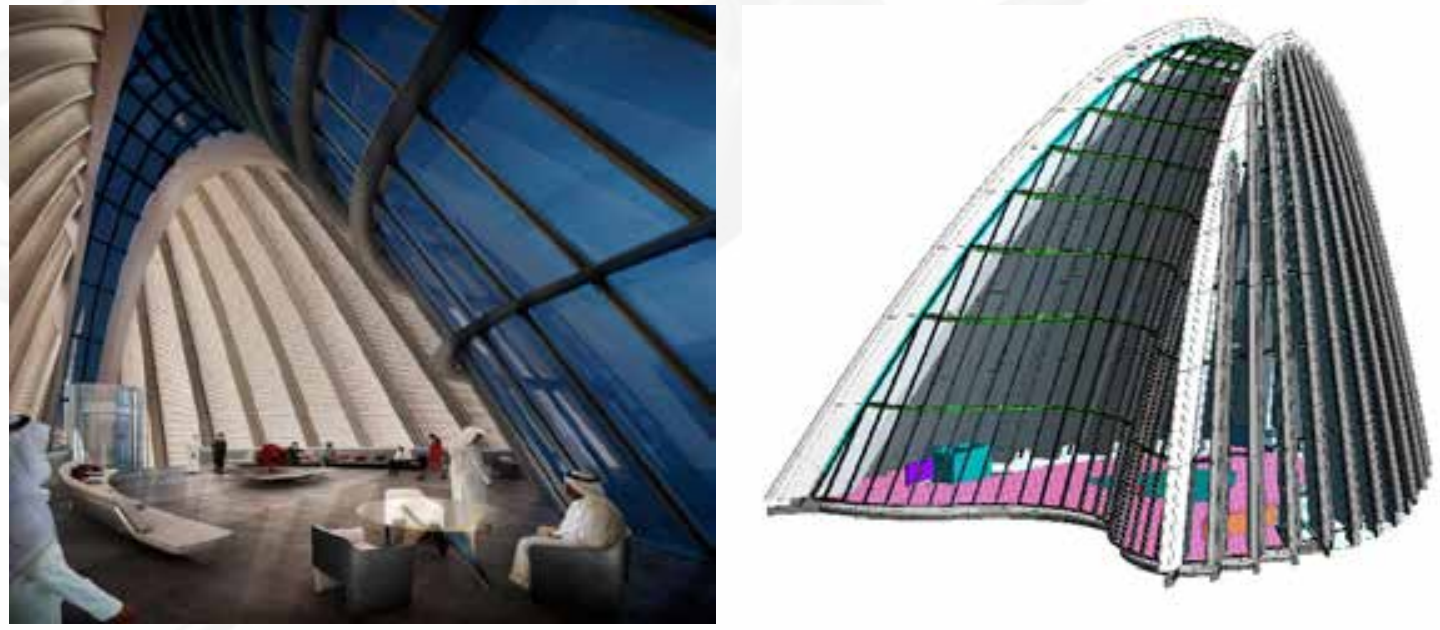

Figure 05: NBK Kuwait peak lobby render image and 3D modelling.
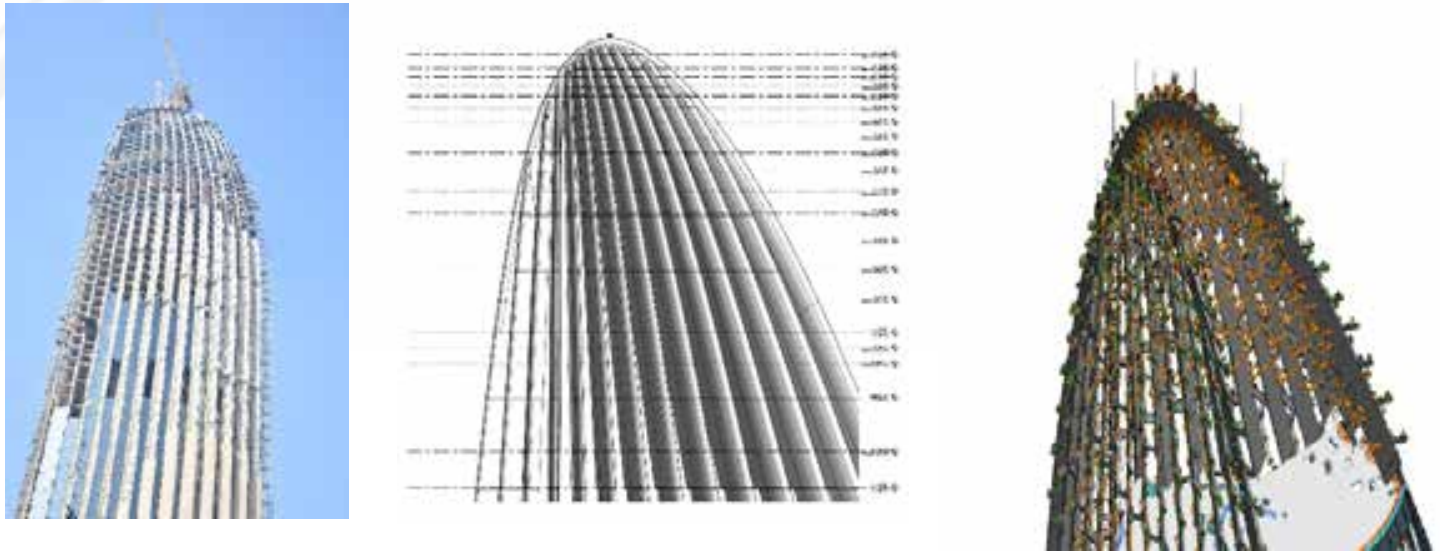

Figure 06: The complex geometry creation and fabrication modeling. 

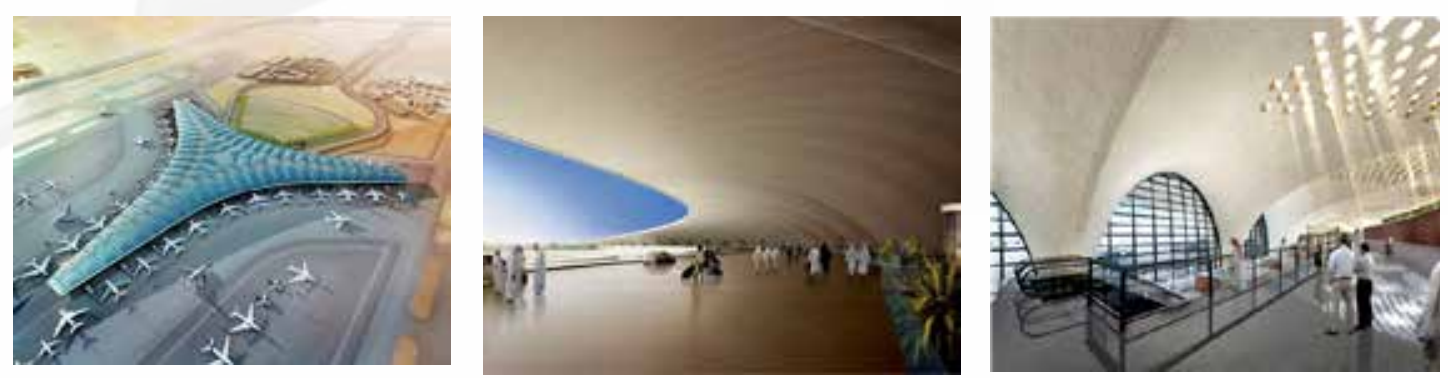

Figure 07: Three-dimensional renders of the new Kuwait Airport T2 terminal, Kuwait City.
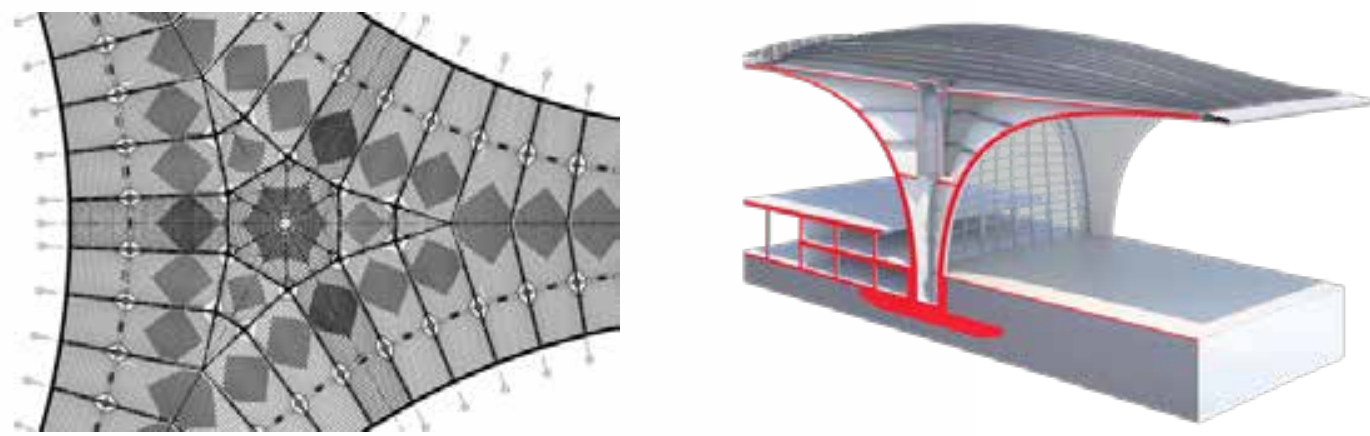

Figure 08: Structural conceptual modeling of the main structure.
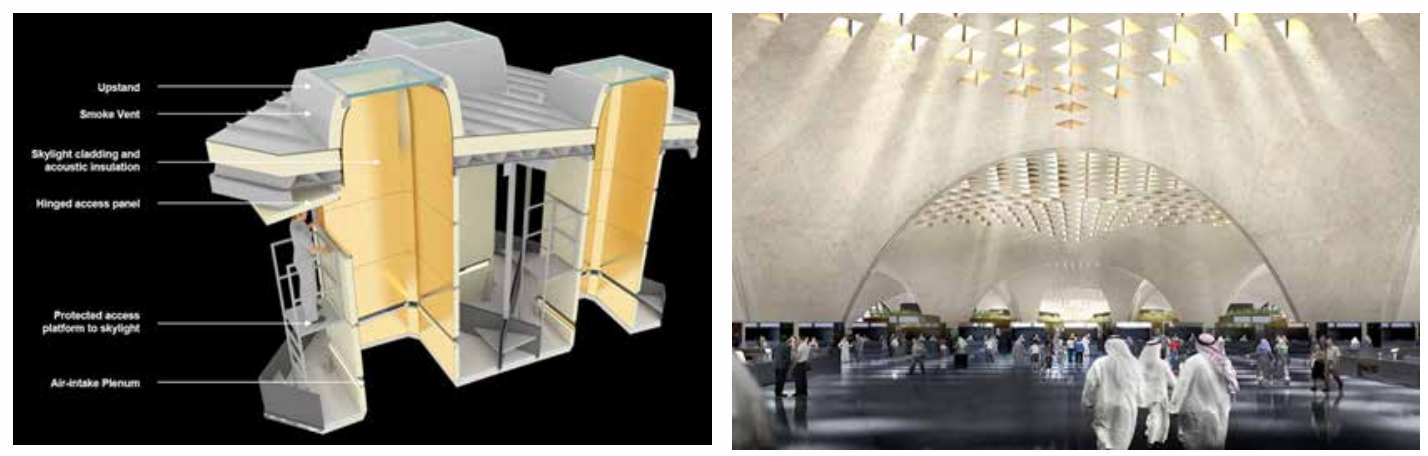

Figure 09: Day light penetration through roof.
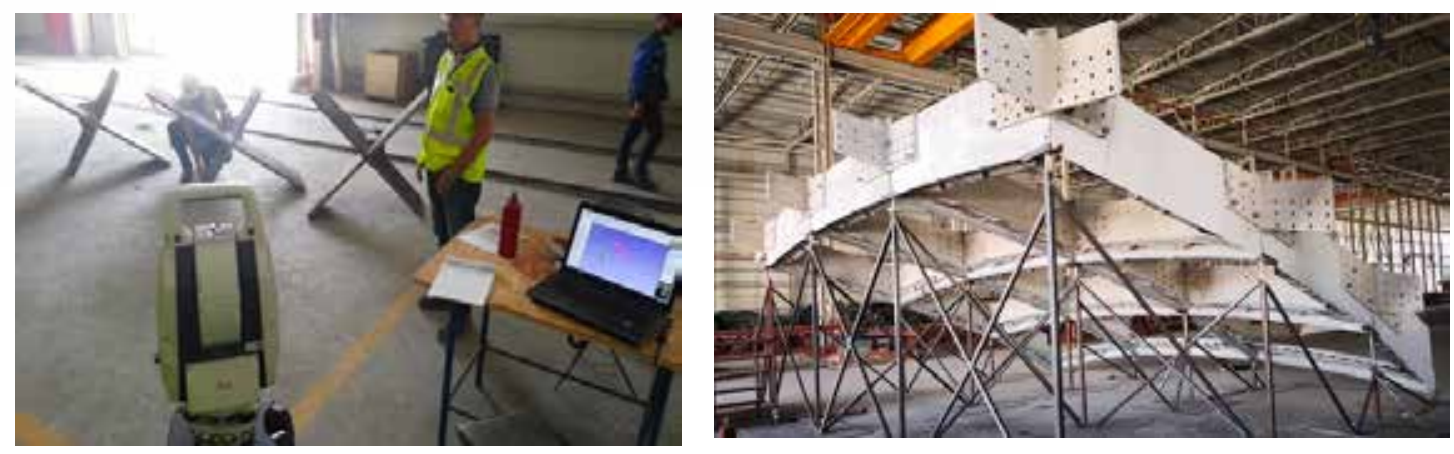

Figure 10: Shell cassettes sub- structure modeling and fabrication. 


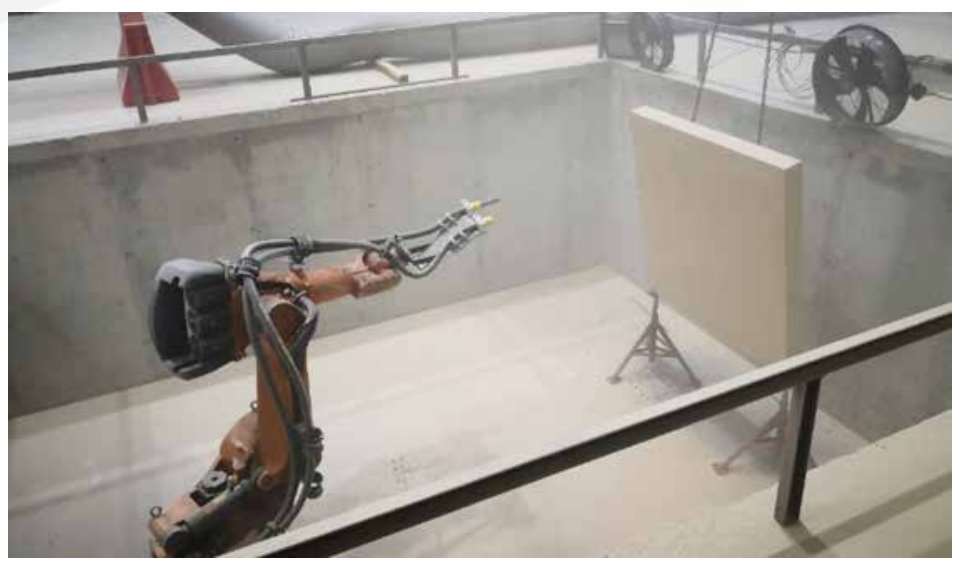

Figure 14: Robotic industrial arm sand blasting shell cassettes for correct finish texture.

\section{Conclusion}

This paper tries to rethink the current architectural and urban morphologies. In recent years, the reach and influence of computational design has tended to be traced on heuristic parametric approaches dealing with numerous normally-considered convergent issues related to design, sociocultural, economic, and environmental issues as reflected by Michael Weinstock 2010, where he pushes the envelope of the current notion of the built environment, and considers the city as a dynamic complex system. He places emphasis on the interactions and connectivity of the flows through its infrastructures, and of the feedbacks and critical thresholds that drive the emergence of new spaces and urban morphologies that are animated by new modalities of culture.

It is also very much apparent how new technologies such as computational simulation and multiscale analytic modelling are facilitating this approach, revealing a new sense of the city as one that is integrated into a complex network of flows and processes, and as much about physical as cultural properties. Running counter to the main theme of the issue is Colin Fournier's energetic counterpoint that seeks to question any assumptions about whether we need such high level precision and zerotolerance in building construction? It also seeks to question if this emergent technology is necessary or is it mere eroticization of technology. Branko Kolarevic (2004) reminds us of the attitude of the building industry and the 'messy' realities of the construction site that design parameters are imported into.

\section{Bibliography}

Castles, H (2014). High-Definition: Zero Tolerance in Design and Production. Architectural Design, Vol 2014.

Heylighen, F (1990). Self-organization, Emergence, and the Architecture of Complexity. Transdisciplinary Research Group, Free University of Brussels, Pleinlaan 2, B-1050 Brussels, Belgium.

Heylighen, H (1983). Synergetics: Non-Equilibrium Phase Transitions and Self-Organization in Physics, Chemistry and Biology. 3rd ed., Springer, Berlin.

Heylighen, F (1989). Building a Science of Complexity. Proceedings of the 1988 Annual Conference of the Cybernetics Society, London.

Heylighen, F (1989). Coping with Complexity: concepts and principles for a support system, 'Proceedings of the conference "Support, Society and Culture", R. Glanville \& G. deZeeuw (eds.) OOC, University of Amsterdam.

Sassen, S (1991). The Global City: New York, London, Tokyo. Princeton University Press, Princeton, NJ.

Shane, D (1976). Urban Design Since 1945: A Global Perspective, John Wiley \& Sons Chichester, p. 29. 
Simon, H. A (1962). The Architecture of Complexity, Proceedings of the American Philosophical Society 106, reprinted 1962.

Simon, H. A (1981). The Sciences of the Articial. $2^{\text {nd }}$ ed. MIT Press, Cambridge MA.

Weinstock, M (2010). The Architecture of Emergence: The Evolution of Form in Nature and Civilization. John Wiley \& Sons, Chichester.

Cite this article as: Dashti H., "Robotic Fabrication as Catalysts for Emergent Topologies and Traditions: Nomadic Small Pavilions and Permanent Mega Structures in Kuwait", International Conference on the $4^{\text {th }}$ Game Set and Match (GSM4Q-2019), Doha, Qatar, 6-7 February 2019, https://doi.org/10.29117/gsm4q.2019.0015 